\title{
Excruciating Headache in a Case of Malabsorption Syndrome
}

\author{
Nandini Chatterjee ${ }^{1}$, Brikodar Patary ${ }^{2}$, Krishanko Das $^{3}$, Shantasil Pyne ${ }^{4}$
}

\begin{abstract}
A 19-year-old male presented to us with anasarca and a history of chronic diarrhea. Investigations revealed primary intestinal lymphangiectasia leading to protein losing enteropathy. Patient was doing well for 1 year on a modified diet until he got admitted with a severe headache, vomiting, and papilledema. Complete workup demonstrated cerebral venous thrombosis as a result of deficiency of protein S.

Keywords: Cerebral venous thrombosis, Intestinal lymphangiectasia, Protein losing enteropathy.

Bengal Physician Journal (2019): 10.5005/jp-journals-10070-6116
\end{abstract}

\section{INTRODUCTION}

Cerebral venous thrombosis constitutes $0.5-1 \%{ }^{1}$ of all strokes, and its annual incidence is 3-4 million in the general population. With the advent of newer diagnostic modalities, an increasing number of cases are being diagnosed. However, cerebral venous thrombosis has been documented only in isolated case reports in malabsorption syndrome.

\section{Case Description}

A 19-year-old male presented with insidious swelling of his body for the past 1 year starting from his legs, later involving the abdomen and face. He was being treated as a case of chronic liver disease elsewhere. On enquiry it was found that he had a history of loose stools two to three times a day for the past 10 years. He was nondiabetic and nonhypertensive. There was no past history of jaundice, hematemesis melena, or encephalopathy. No history of alcohol addiction, hepatotoxic drug intake, or blood transfusion was found.

On examination, he had edema, ascites, glossitis, and moderate pallor. General examination was otherwise normal, and other systemic examination were non-contributory.

Investigations revealed low hemoglobin, lymphopenia, low albumin, and globulin levels in serum. Bilirubin, liver enzymes, and prothrombin time and other blood biochemistry tests were normal. Chest X-ray revealed right-sided pleural effusion, and ultrasound showed moderate ascites. Liver, spleen, and portal vein were normal and so was electrocardiogram (ECG). Urine was normal while stool was negative for occult blood fat or parasites. A protein losing enteropathy was suspected and hence upper gastrointestinal (Gl) endoscopy and colonoscopy were done. Upper Gl endoscopy showed bowel wall edema and inflammation. Biopsy revealed dilated lymphatic channels with chronic inflammatory cells and mucosal edema.

The patient was put on a diet of medium-chain fatty acids (MCFA), vitamins, and diuretics. Albumin and blood were transfused. Patient improved considerably with resolution of edema and ascites. He was lost to follow-up.

On his next admission, he presented with severe headache, vomiting, and drowsiness. Examination revealed bilateral sixthnerve palsy and papilledema. CT scan of the brain and cerebrospinal fluid study were normal. However, magnetic resonance imaging (MRI) of the brain with magnetic resonance venogram (MR venogram) were ordered on clinical suspicion. This clinched the diagnosis of cerebral venous thrombosis. There were no brain

\begin{abstract}
${ }^{1-4}$ Department of Medicine, Institute of Post Graduate Medical Education and Research and Seth Sukhlal Karnani Memorial Hospital, Kolkata, West Bengal, India

Corresponding Author: Brikodar Patary, Department of Medicine, Institute of Post Graduate Medical Education and Research and Seth Sukhlal Karnani Memorial Hospital, Kolkata, West Bengal, India, Phone: +918145005804, e-mail: rivuc23092006@gmail.com

How to cite this article: Chatterjee N, Patary B, Das K, et al. Excruciating Headache in a Case of Malabsorption Syndrome. Bengal Physician Journal 2019;6(2):31-32.
\end{abstract}

Source of support: Nil

Conflict of interest: None

parenchymal changes. MR venogram documented absence of flow signal in superior and inferior sagittal sinus, straight and both transverse sinuses (Figs 1 and 2). Workup for etiology revealed normal prothrombin time (PT), activated partial thromboplastin time (aPTT), antiphospholipid antibody, antithrombin 111, homocysteine, and protein C levels (65-135 IU/ML) and function. The protein $\mathrm{S}$ level found to be $35 \mu \mathrm{g} / \mathrm{dL}(70-140 \mu \mathrm{g} / \mathrm{dL})$, was significantly diminished. He was put on heparin and warfarin. His headache disappeared and vision improved.

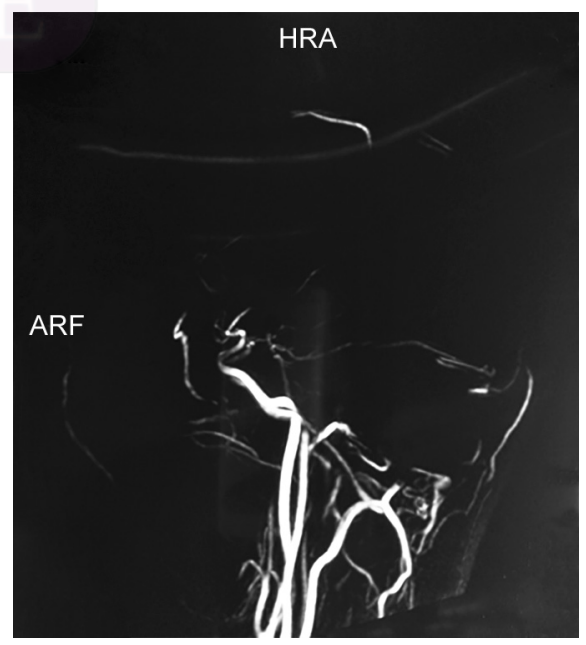

Fig. 1: Contrast-enhanced magnetic resonance venogram shows absence of flow signal in superior and inferior sagittal sinus, straight and both transverse sinuses in sagittal view 


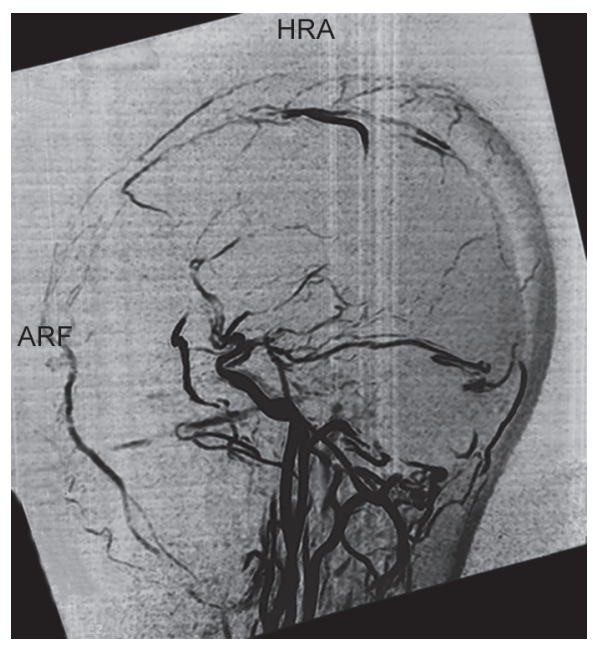

Fig. 2: Digital subtraction angiography (DSA) images reflect thrombosis of superior sagittal, transverse, and straight sinuses

\section{Discussion}

The incidence of cerebral venous thrombosis is now said to be much more in the Indian subcontinent than thought before. The entity is often missed in conventional CT brain. But novel imaging techniques have results in greater accuracy of diagnosis.

The most common cause of cerebral venous thrombosis is the presence of a prothrombotic state either inherited or acquired. Conventionally it is more common in young females. Our patient is a young boy who had a deficiency of protein S attributed to longstanding protein-losing enteropathy. Nephrotic syndrome has often been incriminated in predisposing to thrombosis.

The pathophysiology of thrombosis in protein losing enteropathy (PLE) has been the study of disease-related hemostatic derangements. Because of the loss of albumin, various important hemostatic proteins of similar sizes are also lost in the stools. Prominent among these are the loss of important coagulation regulatory proteins, including antithrombin and protein $\mathrm{S}^{2}{ }^{2}$ Counterbalancing these losses is the synthetic rate at which the liver (primarily) produces hemostatic proteins like fibrinogen. As a result, there is a net shift in the hemostatic balance toward a prothrombotic milieu. These alterations are thought to be the primary mechanism that produces the increased risk of thromboembolism in patients with protein-losing enteropathy. Similar pathogenesis has been proposed in nephrotic syndrome (NS). 3,4

No studies are present on cerebral venous thrombosis (CVT) in malabsorption. However, certain case reports have documented DVT and PE in ulcerative colitis with protein-losing enteropathy. ${ }^{5}$ Mesenteric vein thrombosis associated with systemic lupus erythematosus (SLE) enteritis has also been reported. ${ }^{6}$ Angiography revealed occlusion in a peripheral branch of the superior mesenteric vein. Furthermore, both the patient and his son had low protein $C$ levels. Histologic examination of the resected ileum revealed multiple erosions and submucosal fibrosis with organized venous thrombi in the mesenteric veins.

An inherited syndrome (CHAPLE) with CD55 deficiency showed multiple thromboses in the mesenteric and hepatic veins, heart, and cerebral veins that were associated with an intracranial hemorrhage. associated with protein-losing enteropathy, lymphangiectasia, and malnutrition. The natural history of the disease includes early death related to severe thrombotic events. CD55 deficiency test was negative in our patient. ${ }^{7}$

Our patient developed another problem as we started him on warfarin and heparin therapy. In a very short time his prothrombin time overshot the recommended target range probably due to the defect in his clotting factor dynamics and his warfarin had to be titrated slowly with care to achieve target international normalized ratio (INR).

To conclude, cerebral venous thrombosis is an emerging important cause of stroke in both genders, occurring in a wide variety of comorbidities and to be kept in mind for early diagnosis and intervention.

\section{References}

1. Dash D, Prasad K, Joseph L. Cerebral venous thrombosis: an Indian perspective. Neurol India 2015;63(3):318-328. DOI: 10.4103/00283886.158191.

2. Greenblatt HK, Nyugen BK. Ménétrier's disease presenting as recurrent unprovoked venous thrombosis: a case report. J Med Case Rep 2019;13(1):14. DOI: 10.1186/s13256-018-1952-0.

3. Llach F. Thromboembolic complications in nephrotic syndrome. Coagulation abnormalities, renal vein thrombosis, and other conditions. Postgrad Med 1984;76(6):111-114. DOI: 10.1080/00325481.1984.11698782.

4. Hanevold CD, Lazarchick J, Constantin MA, et al. Acquired free protein $S$ deficiency in children with steroid resistant nephrosis. Ann Clin Lab Sci 1996;26(3):279-282.

5. Kawaguchi Y, Mine T, Kawana I, et al. Protein-losing enteropathy, deep venous thrombosis and pulmonary embolism in a patient with generalized inflammatory polyposis in remission stage of ulcerative colitis. Clin J Gastroenterol 2009;2(3):156-160. DOI: 10.1007/s12328008-0060-x.

6. Lertnawapan R, Sakonlaya D. Lupus protein-losing enteropathy patient with protein $\mathrm{C}$ and protein $\mathrm{S}$ deficiency-induced thrombosis: a case report with review of the literature. Acta Reumatol Port 2017;42(3):265-268.

7. Ozen A, Comrie WA, Ardy RC, et al. CD55 deficiency, early-onset protein-losing enteropathy, and thrombosis. N Engl J Med 2017;377(1):52-61. DOI: 10.1056/NEJMoa1615887. 